\title{
Modélisation des lasers à excimères excités par décharge
}

\author{
J. Bretagne* et E. Estocq*;**
}

* Laboratoire de Physique des Gaz et Plasmas, URA 73 du CNNRS, Université Paris-Sud, 91405 Orsay Cedex, France

** Société SOPRA, 26 rue Pierre Joigneaux, 92270 Bois-Colombes, France

Abstract.

A self-consistent model for the discharge-excited excimer laser is developed. The model is applied to the xenon-chloride laser. We simultaneously solve the time-dependent Boltzmann equation for the electron energy distribution function coupled to the kinetic equations for the densities of excited and ionized species and to the electrical circuit equations. A particular attention is devoted to the $\mathrm{HCl}(X, v)$ vibrational distribution and to its consequences to the dissociative attachment of $\mathrm{HCl}$. Some comparisons of the model with experimental results are discussed.

\section{INTRODUCTION.}

Les lasers à excimères fonctionnent à partir de l'émission d'une molécule dimère excitée dont l'état fondamental correspondant est dissociatif ou très faiblement lié. Ils présentent donc l'avantage de permetire une inversion de population quasi-automatique. Nous distinguons l'appellation excimère dans le cas où les deux atomes sont identiques de celle d'exciplexe où les deux atomes sont de nature différente. La terminologie anglo-saxonne ne distingue pas les deux cas et utilise le terme générique d'excimère. Nous nous intéressons en fait au deuxième cas et plus précisément à celui des halogénures de gaz rares et à l'exemple du laser Xénon-Chlore.

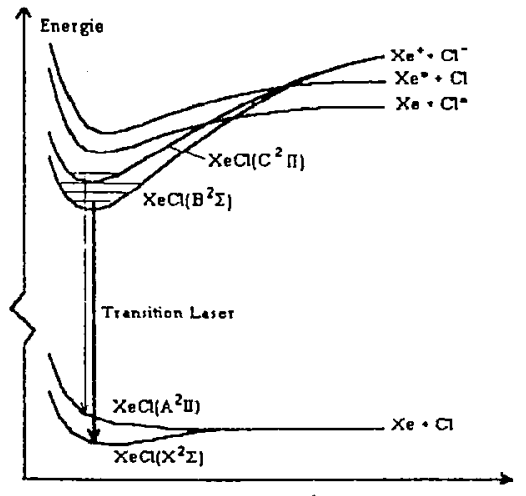

Distence internucléaize

Figure 1. Diagramme schématique d'énergie de la molécule $\mathrm{XeCl}$.

HCl.

Les conditions typiques de fonctionnement du laser sont les suivantes: pression comprise entre 2 et 10 atmosphères, mélange de gaz constitué d'environ $99 \%$ de néon, $1 \%$ de xénon et $0,1 \%$ d'acide chlorhydrique. La pression étant élevée, il est nécessaire 
d'appliquer une préionisation afin d'obtenir une ionisation homogène du milieu laser et d'éviter que la décharge ne dégénère en arc. Une densité d'électrons de préionisation de l'ordre de $10^{8} \mathrm{~cm}^{-3}$ permet d'assurer une bonne homogénéité de la décharge. Cette préionisation peut être effectuée préalablement à l'application de la tension entre les électrodes ou bien, la tension étant établie, une injection brève d'électrons provoque le démarrage de la décharge, c'est la technique plus récente du photodéclenchement ${ }^{[2]}$. La préionisation est généralement produite soit par le rayonnement U.V. d'une décharge annexe, soit par des rayons $X$ engendrés par impact d'un faisceau d'électrons d'énergie comprise entre 50 et $100 \mathrm{keV}$ sur une feuille mince de matériau lourd (tantale, or).

\section{UN MODELE DE DECHARGE LASER.}

Un des objectifs de la modélisation est de permettre de déterminer les espèces excitées et ionisées importantes et les voies de formation de l'état exciplexe $\mathrm{XeCl}^{*}$ qui donne lieu à l'émission laser. Dans la décharge, la fonction de distribution en énergie des électrons est non-maxwellienne, le modèle doit nécessairement la calculer en tenant compte de son couplage avec les espèces présentes dans le milieu. De plus, le laser fonctionne en impulsion, on doit considérer le couplage entre le circuit d'alimentation et décharge: son impédance varie rapidement et constitue un élément important du circuit électrique.

Un des problèmes importants, encore controversé, de la modélisation du laser $\mathrm{XeCl}$ est celui de l'excitation vibrationnelle de la molécule $\mathrm{HCl}$ dans l'état électronique fondamental $\mathrm{X}$ : dans les gaz électronégatifs, on a en général une influence très importante de l'excitation vibrationnelle sur les taux effectifs d'attachement dissociatif.

Dans le modèle développé ici on suppose le milieu laser homogène: ce n'est qu'une approximation. Des modèles à une ou deux dimensions, nécessairement plus simples du point de vue de la cinétique permettent d'étudier le problème des inhomogénéités du milieu qui sont cause des instabilités de fonctionnement de ce laser.

\subsection{Equation de Boltzmann.}

La f.d.e.e. $n(\epsilon, t)$ est déterminée à l'aide de l'équation de Boltzmann dépendant du temps, $n(\epsilon, t)$ représente la densité d'électrons par unité de volume et par unité d'intervalle d'énergie. Si nous supposons que le plasma est homogène, $n(\epsilon, t)$ est déterminée $\operatorname{par}^{[3-5] \text { : }}$

$$
\frac{\partial n(\epsilon, t)}{\partial t}=\frac{\partial J_{F}(\epsilon)}{\partial \epsilon}+\frac{\partial J_{e-n}(\epsilon)}{\partial \epsilon}+\frac{\partial J_{e-e}(\epsilon)}{\partial \epsilon}+K_{i o n}+K_{e x c}+P_{r e c}+S(\epsilon)
$$

où les termes en $\partial J(\epsilon) / \partial \epsilon$ représentent respectivement le flux d'électrons dans

l'espace des énergies dû au chauffage par le champ électrique appliqué (F), le terme correspondant aux collisions élastiques électron-atome ou électron-molécule (e-n), le terme d'échange d'énergie entre les électrons due aux collisions coulombiennes (e-e). $\mathrm{K}_{\text {iun }}$ correspond au flux d'électrons dû aux processus d'ionisation, $\mathrm{K}_{\mathrm{exc}}$ est le terme correspondant aux collisions inélastiques et superélastiques, $\mathbf{P}_{\text {rec }}$ est le terme de perte dû à la recombinaison ou à l'attachement dissociatif. Enfin, $S^{\prime}(\epsilon)$ est un terme source qui correspund à la production d'électrons par la préionisation, par collisions Penning et par ionisation associative.

L'équation de Boltmann est couplée à l'évolution des densités d'espèces excitées et ionisées par les termes de collisions, essentiellement les termes d'ionisation, de collisions inélastiques et superélastiques, de recombinaison et de collisions Penning. L'importance du terme de collisions e-e dépend de le densité électronique.

La méthode que nous utilisons ${ }^{[4,5]}$ pour calculer la f.d.e.e. est dérivée de la méthode de Rockwood ${ }^{[3]}$. La f.d.e.e $n(\epsilon, t)$ est discrétisée sur une grille de cellules élémentaires $\left[\epsilon_{i}-\omega_{i} / 2\right.$ ,$\left.\epsilon_{i}+\omega_{i} / 2\right]$ avec $i \in[1, N], N$ étant le nombre total de points de la grille. Les largeurs $\omega_{i}$ des intervalles d'énergie sont prises en général croissantes sujvant une progression géométrique 
$\omega_{i}=\eta \omega_{j-1}, \eta$ prenant des valeurs typiquement comprises entre 1 et 1,1 . Cette procédure permet d'avoir une bonne précision pour la f.d.e.e. dans le domaine des basses énergies et une précision qui s'avère suffisante pour des énergies plus élevées.

\subsection{Espèces considérées et équations cinétiques.}

Les densités $\mathbf{N}_{\mathbf{k}}$ des espèces excitées et ionisées sont régies par un ensemble d'équations bilan (équations cinétiques ou maitresses) qui tiennent compte de l'ensemble des processus dans lesquels sont impliquées ces espèces. Parmi ces processus, il faut, bien entendu, inclure toutes les collisions électroniques qui intervienneir dans l'équation de Boltzmann et qui produisent un changement d'état ou d'énergie interne des espèces (ionisation, excitation et désexcitation, recombinaison,...).

Il faut aussi tenir compte des processus radiatifs (émission spontanée, absorption de rayonnement, émission stimulée), des collisions entre espèces lourdes qui seront essentiellement des collisions à deux corps et trois corps, importantes en raison des pressions élevées.

On tient compte de l'émission stimulée par une équation relative à la densité de photons laser dans la décharge. Cette densité de photons, couplée aux équations maîtresses, est traitée comme une équation cinétique. Les processus de photoexcitation et de photoionisation par le rayonnement laser sont inclus dans le modèle.

L'écriture de ces équations passe par une étape préalable qui consiste à établir un schéma cinétique. Les principaux états effectifs dont nous tenons compte pour les équations maîtresses sont les suivants ${ }^{[6]}$ :

- pour le néon: le niveau fondamental $\mathrm{Ne}(2 \mathrm{p}), 4$ niveaux excités atomiques effectifs [Ne(3s), $\mathrm{Ne}(3 \mathrm{p}), \mathrm{Ne}(3 \mathrm{~d}, 4 \mathrm{~s})$ et $\mathrm{Ne}(4 \mathrm{p})], 2$ états moléculaires excimères $\left[\mathrm{Ne}_{2}\left({ }^{3} \Sigma\right)\right.$ et $\left.\mathrm{Ne}_{2}\left({ }^{1} \Sigma\right)\right]$, l'ion atomique $\mathrm{Ne}^{+}$et l'ion moléculaire $\mathrm{Ne}_{2}{ }^{+}$.

- pour le xénon: le niveau fondamental $\mathrm{Xe}(5 \mathrm{p}), 6$ niveaux excités atomiques effectifs $\left[\mathrm{Xe}(6 \mathrm{~s}), \mathrm{Xe}\left(6 \mathrm{~s}^{\prime}\right), \mathrm{Xe}(6 \mathrm{p}), \mathrm{Xe}\left(6 \mathrm{p}^{\prime}\right), \mathrm{Xe}(5 \mathrm{~d})\right.$ et un groupe d'états correspondant aux configurations $7 \mathrm{~s}, 7 \mathrm{p}, 6 \mathrm{~d}$ et $4 \mathrm{f}], 2$ états moléculaires excimères $\left[\mathrm{Xe}_{2}\left({ }^{3} \Sigma\right)\right.$ et $\left.\mathrm{Xe}_{2}\left({ }^{1} \Sigma\right)\right]$, l'ion atomique $\mathrm{Xe}^{+}$et l'ion moléculaire $\mathrm{Xe}_{2}{ }^{+}$.

- pour $\mathrm{HCl}$ et ses états dérivés: nous avons introduit 7 niveaux vibrationnels $\mathrm{HCl}(\mathrm{X}, \mathrm{v}), \mathrm{v}=0$ à 6 , les espèces $\mathrm{H}, \mathrm{Cl}$, l'ion négatif $\mathrm{Cl}^{-}, \mathrm{HCl}^{+}$et un état électronique $\mathrm{HCl}^{*}$ dissociatif.

- pour les complexes formés à partir des 3 composants de base: 2 états excités NeXe* et $\mathrm{NeXe}^{* *}$, l'ion moléculaire $\mathrm{NeXe}^{+}$et les états exciplexes excités $\mathrm{XeCl}^{*}$ et $\mathrm{Xe}_{2} \mathrm{Cl}^{*}$. Nous avons tenu compte de l'état $\mathrm{XeCl}(\mathrm{X})$ faiblement lié, état qui peut limiter l'inversion de population et la puissance laser.

Pour la molécule $\mathrm{HCl}$, nous avons inclus une cinétique complète qui tient d'un nombre plus élevés d'états vibrationnels que les modèles habituels qui se limitent généralement à 3 ou 4 états. De plus nous avons, à partir des travaux de Gorse et $\mathrm{al}^{[7,8]}$, considéré un ensemble de processus tels que les échanges $\mathrm{V}-\mathrm{V}$ et $\mathrm{V}-\mathrm{T}$ et la dissociation directe de $\mathrm{HCl}$ qui ne sont pas considérés dans les modèles habituels.

Les populations $\mathbf{N}_{\mathbf{v}}$ des états vibrationnels $\mathrm{HCl}(X, v)$ sont régies par un système d'équations qüis s'écrit:

$$
\begin{aligned}
& \frac{d N_{v}}{d t}=\left(\frac{d N_{v}}{d t}\right)_{e-V}+\left(\frac{d N_{v}}{d t}\right)_{V-V}+\left(\frac{d N_{v}}{d t}\right)_{V-T} \\
&+\left(\frac{d N_{v}}{d t}\right)_{\text {e-ad }}+\left(\frac{d N_{v}^{\prime}}{d t}\right)_{\text {dis }}+\left(\frac{d N_{v}}{d t}\right)_{\text {rec }}+\left(\frac{d N_{v}}{d t}\right)_{\text {rad }}
\end{aligned}
$$

Les différents termes de l'équation (2) représentent respectivement:

- la production et la destruction d'états vibrationnellement excités par collision 
électronique (processus e-V):

$$
e+H C l(v)=\mathrm{HCl}^{-}=e+\mathrm{HCl}(w)
$$

- la redistribution des quanta vibrationnels par les processus $V-V$ :

$$
\mathrm{HCl}(v)+\mathrm{HCl}(w)=\mathrm{HCl}\left(v^{\prime}\right)+\mathrm{HCl}\left(w^{\prime}\right)
$$

- leur destruction par les processus de désactivation vibration-translation (V-T):

$$
H C l(v)+M-H C l(v)+M \quad v>v^{\prime}
$$

où $\mathbf{M}$ est un atome ou une molécule présent dans la décharge; dans ce processus les atomes $\mathrm{H}$ et $\mathrm{Cl}$ sont particulièrement actifs.

- l'attachement dissociatif (e-ad) qui conduit à la formation de l'ion $\mathrm{Cl}$ :

$$
\mathrm{e}+\mathrm{HCl}(\mathrm{v}) \rightarrow \mathrm{HCl}^{-}-\mathrm{H}+\mathrm{Cl}^{-}
$$

- la dissociation par impact électronique (dis):

$$
e+H C l(v)-e+H+C l
$$

- la recombinaison (rec), soit par réassociation à trois corps:

$$
H+C l+M \rightarrow H C l(v)+M
$$

où $\mathrm{M}$ est le troisième corps, soit par détachement associatif:

$$
\mathrm{H}+\mathrm{Cl}^{-} \rightarrow \mathrm{HCl}+e
$$

- enfin, les désexcitations radiatives (rad) des vibrations.

Le modèle cinétique nécessite la connaissance d'un grand nombre de données: taux de réactions à 2 et 3 corps, probabilités de transitions radiatives, sections efficaces de collisions électroniques,... Certaines de ces données peuvent être extraites de la litérature mais beaucoup sont inconnues ou incertaines. Pour les valeurs qui sont en pratique utilisées, il faudra effectuer des tests de sensibilités des résultats du modèle à ces valeurs et s'appuyer, chaque fois que c'est possible sur des résultats expérimentaux.

Les sections efficaces (collisions élastiques, excitations, ionisations) pour le néon et le xénon sont pour l'essentiel celles données par Puech et Mizzi ${ }^{[9]}$. Les sections efficaces pour $\mathrm{HCl}$ et les constantes des réactions $\mathrm{V}-\mathrm{V}, \mathrm{V}-\mathrm{T}$ relatives à $\mathrm{HCl}(\mathrm{V})$ sont celles utilisées par le groupe de $\mathrm{Bari}^{[8]}$. Les sections efficaces d'attachement dissociatif sont celles données par Teillet-Billy et Gauyacq ${ }^{(10)}$. Les constantes de réactions à 2 et 3 corps sont pour l'essentiel celles utilisées par Kannari ${ }^{[1]}$.

\subsection{Couplage de la décharge laser au circuit électrique.}

Sans détailler le circuit électrique d'alimentation de la décharge qui peut varier d'un dispositif à un autre, considérons que $I_{d}$ et $V_{d}$ sont respectivement le courant et la tension appliquée à la décharge. Le problème est de coupler les variables électriques macroscopiques du circuit électrique aux paramètres microscopiques du plasma. Si nous faisons l'hypothèse que le plasma est homogène et purement résistif, on écrit, d'un point de vue microscopique, que la densité de puissance $P$ déposée par le champ appliqué est donnée formellement par:

$$
P=(E / N)^{2} \cdot J_{E}
$$

où $\mathrm{J}_{E}$ représente le flux total d'électrons dans l'espace des énergies dû au champ électrique. Ce terme s'exprime à l'aide de $J_{F}(\epsilon)$ qui intervient dans l'équation de Boltzmann (1) et donc en fonction de la f.d.e.e. $n(\epsilon, t)$.

Du point de vue macroscopique, si $\mathbf{S}$ est la section de la décharge et $\mathbf{I}$ la distance interélectrodes, P s'exprime par: 


$$
P=I_{d} V_{d} /(S l)=I_{d} E / S
$$

L'utilisation des relations (3) et (4) permet d'exprimer E/N, alors variable secondaire, à chaque instant en fonction des paramètres géométriques de la décharge, de $\mathrm{I}_{d}$ (variable indépendante) et de la f.d.e.e. ${ }^{\mid 12 !}$.

\subsection{Le traitement numérique cohérent du modèle.}

La méthode habituelle qui consiste à découpler la résolution du système équation de Boltzmann-équations cinétiques-équations électriques en échelles de temps distinctes pour chacune de ses trois parties risque d'être dangereuse, notamment pour la modélisation des décharges pulsées: soit en phase d'ionisation rapide du plasma, soit en phase de décroissance. Par exemple, une variation rapide du champ réduit $E / N$ ne s'accompagne pas nécessairement d'un équilibre instantané de la f.d.e.e. avec $\mathrm{E} / \mathrm{N}$.

La méthode que nous utilisons, consiste à intégrer le système global de manière simultanée sans avoir recours au découplage en échelles de temps et à l'hypothèse quasistationnaire pour la f.d.e.e. Ceci est facilité par l'utilisation de routines maintenant classiques de résolution de systèmes d'équations différentielles à variations raides (stiff equations) ${ }^{[13]}$. Les avantages sont que le choix du pas de temps d'intégration se fait de manière automatique en fonction des conditions plasmas rencontrées et, surtout, que l'ensenble du système est traité de manière cohérente. L'inconvénient majeur est que, pour obtenir un gain de temps CPU, qui peut être très appréciable, il est en pratique nécessaire de définir explicitement le jacobien du système global.

\section{RESUITTATS.}

\subsection{Résultats du modèle.}

Les résultats présentés ici correspondent à l'utilisation du modèle pour les conditions de fonctionnement d'un laser développé par SOPRA ${ }^{[14]}$. Le circuit électrique comprend deux bancs de condensateurs chargés en parallèle. Ces deux bancs de condensateurs sont connectés en série aux bornes de la décharge : un circuit annexe déclenché par un thyratron permet d'inverser la tension aux bornes de l'un des bancs de condensateurs. Le tension appliquée à la décharge croît alors. Nous donnons les résultats pour une pression totale du gaz de 4 bars et à une composition de $99,2 \%$ de néon, $0,75 \%$ de xénon et $0,075 \%$ de $\mathrm{HCl}$. La tension initiale de charge des condensateurs est de $20 \mathrm{kV}$.

Nous donnons sur la Figure 2, l'évolution typique des paramètres électriques de la décharge. La tension $V_{d}$ aux bormes de la décharge, initialement nulle, commence par croître. Les électrons de préionisation, dont le densité est de l'ordre de quelques $10^{6} \mathrm{~cm}^{-3}$ sont multipliés par le champ appliqué. Lorsque la densité électronique commence à atteindre des valeurs importantes (typiquement supérieures à $10^{12} \mathrm{~cm}^{-3}$ ) le courant de décharge croît brutalement et la tension de décharge chute pour se stabiliser à des valeurs qui correspondent à des champs réduits $\mathrm{E} / \mathrm{N}$ de l'ordre de 1 à $2 \mathrm{Td}$ (phase de plateau) avant de s'inverser. Sur la Fig. 2 nous avons également représenté l'évolution temporelle de la puissance laser.

Nous avons reporté sur la Figure 3 les ailures de la f.d.e.e. réduite, qui est définie par $f(\epsilon, t)=\epsilon^{1 / 2} n(\epsilon, t) / n_{t}$, à différents instants de la décharge. Dans la phase de montée de la tension de décharge, la f.d.e.e. s'étend vers les énergies élevées. En même temps que se développe l'jonisation du milieu, les états excités sont peuplés et la f.d.e.e. commence à présenter un épaulement, visible sur la Fig. 3, qui apparaît dans la queue de la f.d.e.e.. Il est produit par les collisions superélastiques des électrons froids sur les états excités, il est d'autant plus prononcé que le champ réduit $E / N$, et la tension de décharge, sont plus faibles.

Le modèle permet de tester l'importance des différentes espèces dans la production ou la destruction de l'état laser $\mathrm{XeCl}^{*}$. En ce qui concerne les espèces excitées et jonisées provenant du néon, elles jouent un róle très mineur dans la cinétique. Par exemple, les 
densités d'ions $\mathrm{Ne}^{+}$et $\mathrm{Ne}_{2}^{+}$n'atteignent pas $10^{12} \mathrm{~cm}^{-3}$ alors que la densité électronique maximale est de l'ordre de $10^{15} \mathrm{~cm}^{-3}$. Le néon intervient donc uniquement pour déterminer le f.d.e.e. et, bien entendu, comme partenaire dans les collisions à 2 et 3 corps entre particules lourdes. Dans le milieu laser, un modèle sophistiqué pour les niveaux du néon
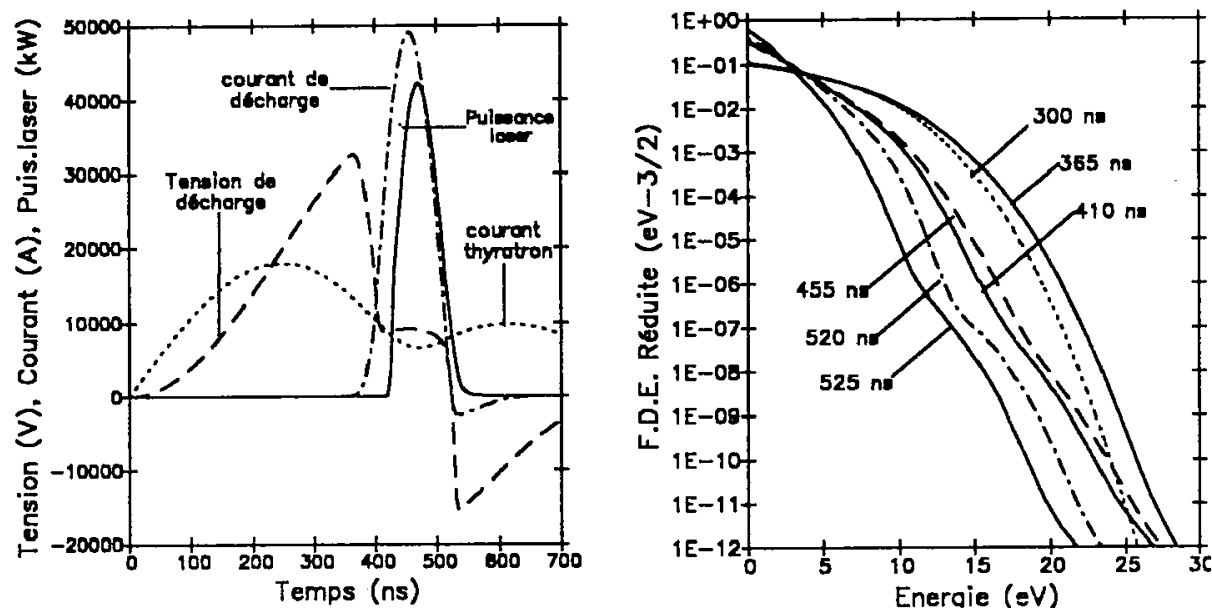

Figure 2. Evolution des paramètres électri- Figure 3 f.d.e.e. réduite à différents instants ques et de la puissance laser dans la décharge. de la décharge.
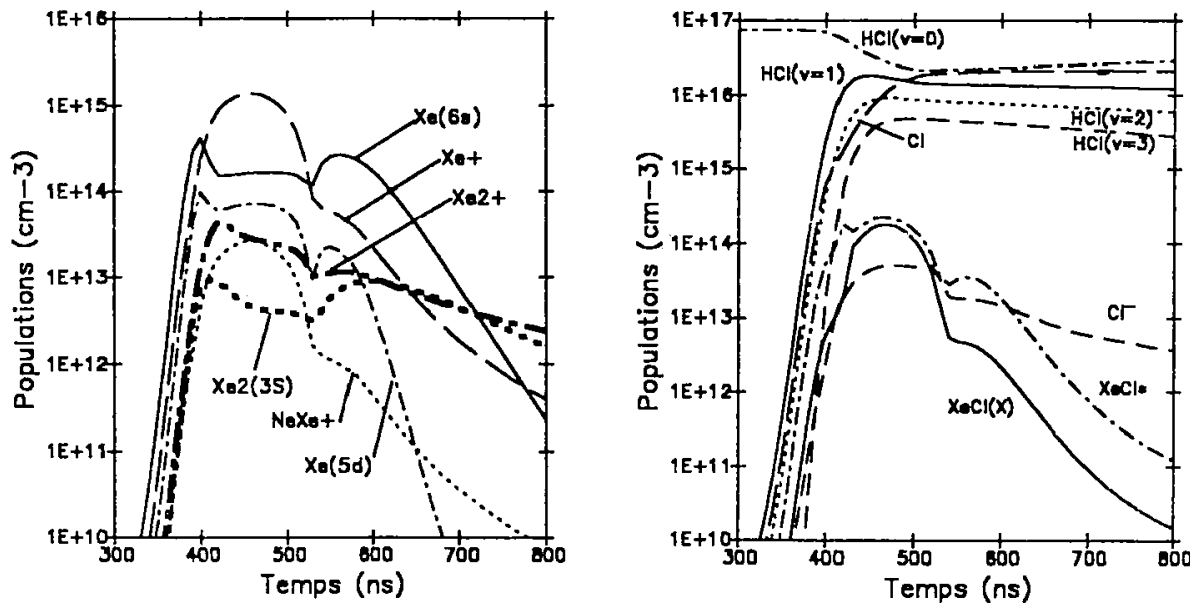

Figure 4. Evolution temporelle des popula- Figure 5. Evolution temporelle des états liés tions de xénon. à $\mathrm{HCl}$.

n'est donc pas nécessaire.

Les densités des états excités les plus peuplés du xénon et les densités des ions positifs majoritaires $\mathrm{Xe}^{+}, \mathrm{Xe}_{2}{ }^{+}$et $\mathrm{NeXe}^{+}$sont reportés sur la Figure 4. L'ion positif dominant est l'ion $\mathrm{Xe}^{+}$sauf dans la post-décharge où c'est $\mathrm{Xe}_{2}{ }^{+}$. Quant aux états excités, l'état effectif majoritaire est $\mathrm{Xe}(6 \mathrm{~s})$; cependant, il ne faut pas négliger les états excités supérieurs qui jouent un rôle important dans la production d'électrons libres. Un bilan des processus d'ionisation dans la phase de plateau de tension indique que ce sont les états excités les plus hauts en énergie qui apportent la contribution la plus importante à l'ionisation. 
Nous avons représenté sur la Figure 5 l'évolution temporelle d'un certain nombre d'états ou d'espèces liées à la présence de $\mathrm{HCl}$ et, en particulier, l'état exciplexe $\mathrm{XeCl}^{*}$. Pour les états vibrationnels $\mathrm{HCl}(\mathrm{X}, \mathrm{v})$, nous n'avons représenté que les populations des quatre premiers niveaux. On constate que les états vibrationnellement excités ne se peuplent de façon significative que dans la phase finale de l'avalanche (à l'apparition du pallier de tension). Dans la post-décharge, les densités de ces états ne décroissent que très lentement: cela indique que les processus de destruction des quanta vibrationnels, en particulier par les processus V-T ne sont pas très efficaces dans les conditions de la décharge laser ${ }^{[8]}$. Il faut aussi remarquer que la densité d'atomes $\mathrm{Cl}$, par ailleurs tres proche de la densité d'atomes $\mathrm{H}$, est relativement élevée à la fin de la décharge. Le processus de dissociation directe de $\mathrm{HCl}$ par impact électronique (dis) contribue pour une part importante à la formation de ces atomes $\mathrm{H}$ et $\mathrm{Cl}$. Du point de vue cinétique, le milieu laser ne retrouve donc que lentement ses conditions initiales. Remarquons enfin que les densités de l'état $\mathrm{XeCl}^{*}$ et de l'ion $\mathrm{Cl}^{-}$ restent de l'ordre de $10^{14} \mathrm{~cm}^{-3}$, l'ion $\mathrm{Cl}^{-}$a une densité, au moment de l'émission laser, qui est d'environ un ordre de grandeur inférieur à la densité de l'ion $\mathrm{Xe}^{+}$avec qui il se recombine pour former $\mathrm{XeCl}^{*}$.

Le modèle permet de tester l'importance des voies de formation de la molécule $\mathrm{XeCl}^{*}$. Nous reportons sur la Figure 6 les contributions relatives de la voie ionique et la voie neutre. Nous observons qu'avant la phase finale de l'avalanche, la voie neutre prédomine alors que pendant cette phase c'est la voie ionique qui est dominante. Ce résultat est à rapprocher de la Fig. 5 et de l'excitation vibrationnelle de $\mathrm{HCl}$ ainsi que de la dépendance de l'attachement dissociatif en fonction de la vibration. Nous avons représenté sur la Figure 7 les valeurs du coefficient effectif d'attachement dissociatif obtenues lorsque l'on considère les sommes partielles des contributions de chacun des niveaux vibrationnels de 0 à $v$. Ce coefficient est défini par la relation:

$$
\overline{K_{a d}}=\left(\sum_{0}^{v} N_{v} K_{a d}(v)\right) /\left(\sum_{0}^{v} N_{v}\right)
$$
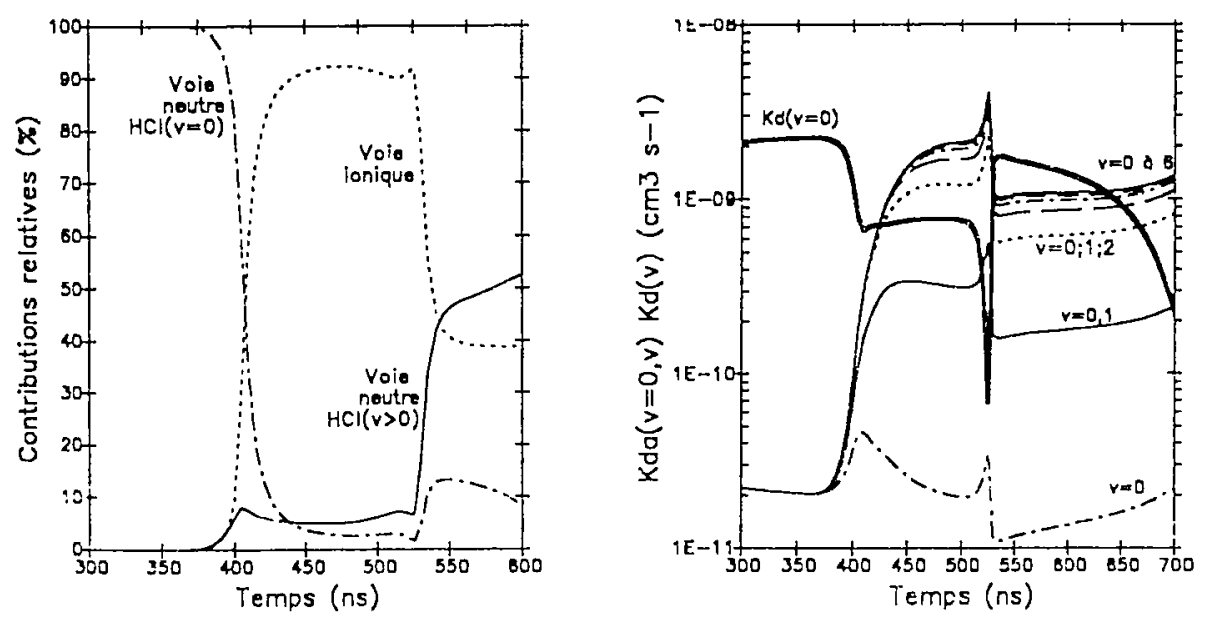

Figure 6. Contributions relatives de la voie Figure 7. Contribution relative des différents jonique et de la voie neutre à la formation de états vibrationn Łels à lattachement dissociatif $\mathrm{XeCl}^{*}$

(voir texte).

Ces sommes partielles ne convergent qu'à partir de $v=4$, ce qui signifie qu'il faut inclure au moins 5 niveaux vibrationnels de $\mathrm{HCl}$ pour obtenir une représentation correcte du 
phénomène d'attachement dissociatif. Le coefficient de dissociation directe $\mathbf{K}_{d}(\mathbf{v}=\mathbf{0})$ est également reporté sur la Fig.7. Nous remarquons que dans la phase de plateau de tension $\mathbf{K}_{d}(\mathbf{v}=\mathbf{0})$ est plus faible que le coefficient effectif d'attachement dissociatif mais $\mathbf{n}$ 'est cependant pas négligeable.

\subsection{Comparaison modèle-expérience.}

Dans l'utilisation du modèle, une étape importante est la comparaison des résultats qu'il donne aux résultats expérimentaux disponibles. Par ailleurs, dans la mesure où de nombreuses données restent incertaines, nous devons tester la sensibilice des résultats à ces données (constantes de réactions, sections efficaces de collisions électroniques,...).

Pour ce qui est des résultats expérimentaux, ceux-ci doivent être diversifiés. Les courant et tension de décharge, la puissance et l'énergie laser extraites constituent des éléments importants mais ne permettent pas de lever la plupart des incertitudes qui subsistent sur les données. Il est nécessaire d'avoir des informations complémentaires sur les principaux niveaux impliqués dans la cinétique de formation de l'état $\mathrm{XeCl}^{*}$. Il convient de s'intéresser en particulier aux états excités du xénon et, en premier lieu, aux états $\mathrm{Xe}^{*}(6 \mathrm{~s})$ et à la distribution virationnelle de $\mathrm{HCl}(\mathrm{X}, \mathrm{v})$. Un élément de cette comparaison est l'étude des temps de retard entre l'application de la préionisation et l'établissement du plateau de tension de décharge: ces temps de retard sont significatifs du dépôt d'énergie, sur les différents états excités et ionisés, à partir des états fondamentaux du néon, du xénon et de $\mathrm{HCl}$. L'accord, pour différents types et conditions de décharge (tension appliquée, pression, composition du gaz,...), entre résuitats expérimentaux et théoriques sont en général bons aussi bien en préionisation classique ${ }^{[6]}$ qu'en photodéclenchement ${ }^{[15]}$.

Cependant, la majeure partie (plus de $90 \%$ ) de l'énergie disponible est déposée dans la phase du plateau de tension pendant laquelle les états excités jouent un rôle important. Des techniques de détermination d'évolution temporelle des densités des états excités Xe*(6s) par absorption du faisceau d'un laser à colorant sonde ont été utilisées mais les interprétations sont délicates en raison de la nécessité de travailler sur les ailes des raies aboutissant à ces niveaux et de bien maîtriser les paramètres d'élargissement et de déplacement des raies sondées ${ }^{[16.17]}$. La distribution vibrationnelle de $\mathrm{HCl}$ qui semble jouer un rôle important pendant le plateau de tension reste un problème ouvert notamment pour ce qui est des états vibrationnels élevés.

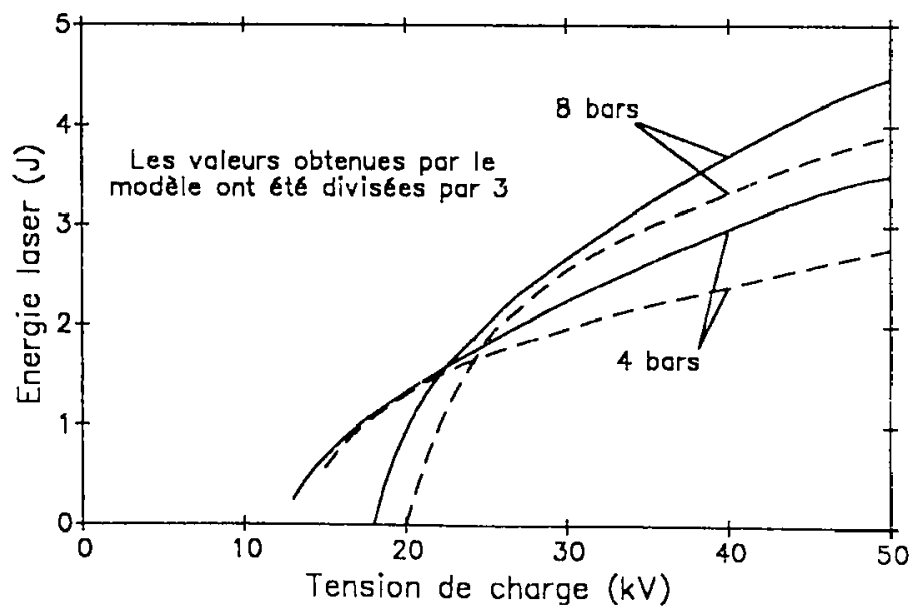

Figure 8. Comparaison des énergies laser extraites calculées (traits pleins) et expérimentales (tirets) en fonction de la tension de charge des condensateurs. 
Nous donnons sur la Figure $\mathbf{8}$, pour deux pressions du mélange gazeux, une comparaison des énergies laser extraites calculées par le modèle et obtenues expérimentalement en fonction de le tension de charge des condensateurs dans le laser SOPRA pour des dimensions de décharge de $4,5 \mathrm{~cm}$ de distance interélectrodes, $4 \mathrm{~cm}$ de largeur et $40 \mathrm{~cm}$ de longueur. Il faut remarquer que les valeurs du modèle ont été divisées par 3. Malgré la bonne concordance entre les allures des courbes, ce facteur 3 qui résulte de la comparaison pose le problème de processus importants non inclus dans le modèle qui limiteraient la puissance et l'énergie laser. Les tests effectués sur différentes données (section efficace d'émission stimulée, sections efficaces d'excitation vibrationnelle de ....., d'attachement dissociatif,...) ne permettent pas jusqu'alors d'expliquer ce facteur.

\section{MODELES A 1 OU 2 DIMENSIONS.}

Une des limites des travaux présentés ici tient au fait que le plasma est considéré comme homogène. Nous n'avons pas présenté ici les travaux qui sont effectués sur les modélisations à une ou deux dimensions et qui permettent d'étudier le problème de l'hétérogénéité de la décharge et le développement des instabilités en liaison par exemple avec une préionisation non homogène de la décharge.

Dans la décharge laser la région cathodique joue un rôle essentiel, elle engendre des ondes de choc qui se propagent dans le milieu gazeux et sont néfastes lors le fonctionnement du laser à taux de répétition élevéli8]. Il est donc important de modéliser cette région. Un modèle auto-cohérent $1 \mathrm{D}$ a été développé par l'équipe de J.P. Boeuf et L. Pitchford ${ }^{[19]}$ permettant de décrire la formation de la région cathodique à partir de charges initiales créées au voisinage de la cathode. Ce modèle de type hydrodynamique (résolution des équations de continuité et de transport de la quantité de mouvement pour les particules chargées) est autocohérent dans le sens qu'il couple les ions, les électrons et le champ électrique dans la région cathodique dont l'extension est supposée être de l'ordre du millimètre. Le corps du plasma est supposé homogène. Les espèces chargées sont supposées être en équilibre local avec le champ électrique.

Les calculs ont permis de déterminer les évolutions temporelles des densités locales d'électrons et d'jons et la répartition du champ électrique au voisinage de la cathode. Ils ont permis d'analyser le passage d'une phase de multiplication des charges qui s'effectue en volume à une phase où la source d'électrons est assurée par l'émission secondaire d'électrons par impact d'ions à la cathode. Il a été ainsi montré que les phénomènes d'émission secondaire à la cathode jouent un rôle fondamental dans le fonctionnement de la décharge. En particulier, la structuration du champ électrique dans la gaine cathodique est fortement dépendante du coefficient d'émission secondaire $\gamma$ du matériau cathodique. Il a également été montré que la région cathodique est le siège d'un échauiffement important origine des ondes de choc engendrées dans la décharge). Cet échauffement pourrait par ailleurs provoquer un chauffage de la cathode et la modification des processus d'émission d'électrons.

L'étude de l'influence de l'inhomogénéité de la préjonisation au voisinage de la cathode ou d'une non-uniformité de l'émission secondaire nécessite l'utilisation d'un modèle à deux dimensions. Un tel modèle est en cours de développement ${ }^{[19]}$. L'étude des phénomènes q::i ont lieu à la cathode ou à sá proximité est certainement une approche ínportante en vue de la compréhension des instaoilités de la décharge laser et de phénomènes de filamentation qui sont observés expérimentalement.

\section{CONCLUSIONS.}

Nous avons présenté ici un modèle auto-cohérent de la décharge du laser xénon-chlore qui, en particulier. inclut une cinétique détaillée des états vibrationnels de $\mathrm{HCl}$. La comparaison des résultats du modèle aux résultats expérimentaux indique un écart important quant à la puissance et l'ćnergie laser et il apparaît que le fonctionnement de ce laser n'est pas encore bien compris du point de v'ue physique. En particulier, des interrogations 
importantes subsistent sur la compréhension du rôle du donneur d'halogène $(\mathrm{HCl})$. Mais il ne faut pas exclure l'existence de processus non pris en compte dans le modèle.

Il faut mentionner qu'une difficulté importante de la modélisation tient au manque de données ou à leur incertitude, notamment pour ce qui a trait aux états vibrationnellement excités $\mathrm{HCl}(\mathrm{X}, \mathrm{v})$ (sections efficaces d'excitation vibrationnelle, d'attachement dissociatif, existence et stabilité des états $v>2, \ldots)$.

Même si un désaccord subsiste entre modèle et expérience, ii reste que les grandes tendances fournies par le modèle et les expériences sont en bon accord. Le modèle - qui est un outıl de travail important pour la compréhension de la physique du mueu laser, la comparaison aux résultats expérimentaux et leur interprétation, l'extrapolation des dispositifs existants - peut facilement être adapté à d'autres systèmes laser, KIF et ArF par exemple, pourvu que l'on puisse disposer des données nécessaires.

Le type d'approche développé ici est complémentaire de modèles à une ou deux dimensions qui sont indispensables si l'on veut étudier le problème des instabilités de la décharge laser. L'étude des phènomènes au voisinage de la cathode est, dans ce cadre, un aspect important.

\section{Remerciements.}

Les auteurs remercient V.Puech, R.Riva et M.Legentil pour les nombreuses discussions à propos de ce travail et J.P.Boeuf, L.Pitchford et leurs collaborateurs de nous avoir communiqué leurs résultats avant publication.

\section{REFERENCES.}

1. Ch.A.Brau, in High-Power lasers and Applications, Ed. K.L.Kompa and H.Walther, Springer-Verlag, Berlin (1978)

2. B.Lacour and M.Vannier, J.Appl.Phys. 62, 754 (1987)

3. S.D. Rockwood, Phys.Rev. A8, 2348 (1976)

4. J.Bretagne, G.Delouya, J.L.Godart and V.Puech, J.Phys.D: Appl. Phys. 14, 1225 (1981).

5. J.Bretagne, J.Godart and V.Puech, J.Phys.D: Appl. Phys. 15, 2205 (1982).

6. E.Estocq, Thèse de doctorat, Université Paris-Sud, Orsay (février 1992)

7. C.Gorse, M.Capitelli, M.Bacal and J.Bretagne, Chem.Phys. 102, 1 (1986)

8. C.Gorse, M.Capitelii, S.Longo, E.Estocq and J.Bretagne, J.Phys.D: Appl.Phys. 24, 1947 (1991)

9. V.Puech and S.Mizzi, J.Phys.D: Appl.Phys. 24, 1974 (1991).

10. D.Teillet-Billy and J.P.Gauyacq, J.Phys.B: At.Mol.Phys. 17, 4041 (1984).

11. F.Kannari, W.D.Kimura and J.J.Ewing, J.Appl.Phys. 68, 2615 (1990)

12. J.Bretagne, Cinétiques couplées dans les les plasmas froids, Ecole de Génie des Procédés Plasmas, Luminy (novembre 1991). A paraître.

13. R.C. Aiken, Stiff computation, Ed. Oxford University, Oxford (1985) et réf. citées.

14. B.Godard, E.Estocq, M.Stehlé, J.Bonnet and D. Pigache, VIII Int. Symp. on Gas Flow and Chemical Lasers (Madrid, 10-14 sept. 1990), SPIE 1397, 59 (1991)

15. M.Legentil, S.Pasquiers, V.Puech and R.Riva, J.Appl.Phys. 72, Août 1992 Sous presse. 16. T.Hammer and W.Bötticher, Appl.Phys.B 48, 73 (1989).

17. M.Legentil, S.Pasquiers, V.Puech and R.Riva, Proc. $11^{\text {th }}$ Int. Conf. on Spectral Line Shapes, Carry-le-Rouet, (8-12 juin 1992).

18. M.L.Sentis, P.Delaporte, B.M. Forestier and B.L.Fontaine, J.Appl.Phys. 66, 1925 (1989)

19. J.P.Boeuf and L.Pitchford, communication privée. 